\title{
Coherent beam stability in the low momentum compaction lattice
}

\author{
S. Heifets and A. Novokhatski \\ Stanford Linear Accelerator Center, Stanford University, Stanford, California 94309, USA
}

(Received 14 February 2006; published 18 April 2006)

\begin{abstract}
The beam dynamics for a quasi-isochronous lattice differs from that in the usual case of a lattice with a large positive momentum compaction factor. In particular, the quasi-isochronous lattice allows us to double the number of bunches which may be an attractive option for colliders. However, microwave instability and, as we show, longitudinal head-tail instability set the threshold for the beam current.
\end{abstract}

DOI: 10.1103/PhysRevSTAB.9.044402

PACS numbers: 29.27.Bd, 29.20.Dh

\section{INTRODUCTION}

Here we consider the coherent stability of bunches in the quasi-isochronous lattice. Study of the coherent beam stability can clarify how useful isochronous lattices are, in particular, for proposed strong longitudinal focusing [1] and, potentially, for increasing the luminosity of a collider such as B-factory with the number of bunches in the ring [2]. The quasi-isochronous lattice allows to have two bunches per rf bucket. Such bunches were observed experimentally [3]. The beam dynamics of two bunches is quite different because the slope of the rf field for two bunches has opposite signs. Therefore, one bunch behaves as a bunch in the lattice with a positive momentum compaction $\alpha$ while the other bunch behaves as a bunch in the lattice with negative $\alpha$. We show that the longitudinal head-tail (LHT) and the microwave instabilities define the threshold of the beam stability. We also study whether the longitudinal feedback (LFB) system may be used to stabilize the LHT instability. Other effects are discussed in [2].

\section{LATTICE DESIGN}

A possible lattice for quasi-isochronous ring was designed before [4]. In Ref. [2] we reproduce the design for the arc cell lattice ignoring the straight sections and matching sections. The optics is shown in Fig. 1.

The momentum compaction calculated with MAD is

$$
\alpha=5.344 \times 10^{-4}+0.05542 \delta-0.0662 \delta^{2} .
$$

Hence, $\alpha_{0}=5.344 \times 10^{-4}, \alpha_{1}=0.0554$, and the energy separation of the stable points $\alpha_{0} / \alpha_{1}$ corresponds to $15.81 \delta_{0}$, and the offset $\Delta x=1.78 \mathrm{~cm}$. Note that $\alpha_{0}$ is smaller than the nominal PEP-II $\alpha_{0}=2.7 \times 10^{-3}$ only by a factor of 5 .

The same lattice can be used to make the lattice isochronous by increasing the strength of a single quadrupole by $2 \%$.

The design of the lattice for the PEP-II low energy positron ring (LER) can be different and based on the lattice with missing bends. The ratio $\alpha_{0} / \alpha_{1}$ in both rings has to be the same to have collisions. We also assume the zero dispersion at the interaction point.

It is also possible to design a lattice where both $\alpha_{0}$ and $\alpha_{1}$ are suppressed [5] and the momentum compaction is dominated by the next term, $\alpha \propto \alpha_{2} \delta^{2}$. The longitudinal phase space in this case can be more complicated. We do not consider such an option here because, generally speaking, the number of stable bunches and their separation in the phase plane become smaller, and the effect of the quantum fluctuation on diffusion between stable points may become the limiting factor for beam dynamics.

\section{LONGITUDINAL DYNAMICS WITH SMALL $\alpha$}

As it is well known [6], longitudinal dynamics is described by the equations

$$
\begin{aligned}
& \frac{d z}{d t}=-c \delta\left(\alpha_{0}+\alpha_{1} \delta\right), \\
& \frac{d \delta}{d t}=\frac{e V \omega_{0}}{2 \pi E_{0}} \cos \left(\phi_{s}-\frac{\omega_{r f} z}{c}\right)-\frac{\omega_{0} U}{2 \pi E_{0}},
\end{aligned}
$$

where $z$ is the shift of a particle from the bunch centroid ( $z>0$ is the shift toward the head of the bunch), $\delta=(E-$ $\left.E_{0}\right) / E_{0}$ is the energy deviation, and $c$ is the velocity of light. Other parameters are the following: $\omega_{0}$ is the revolution frequency, $\phi_{s}$ is the rf phase, $\cos \phi_{s}=U / E_{0}, \alpha=$

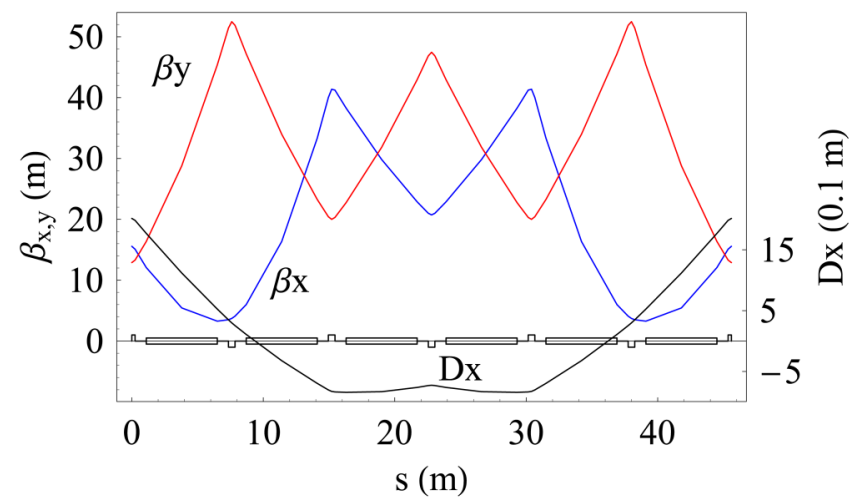

FIG. 1. (Color) Twiss parameters for the quasi-isochronous lattice with $\alpha_{0}=5.344 \times 10^{-4}$. 
$\alpha_{0}+\delta \alpha_{1}$ is the momentum compaction, $E$ is the beam energy, $V$ and $U$ are the rf voltage and the energy loss (including wake field) per turn, respectively.

Equation (2) shows that there are two stable fixed points (FP):

$$
\begin{aligned}
& \text { (1) }\left\{\frac{\omega_{\mathrm{rf}} z_{1}}{c}=0, \delta_{1}=0\right\}, \\
& \text { (2) }\left\{\frac{\omega_{r f} z_{2}}{c}=2 \phi_{s}, \delta_{2}=-\frac{\alpha_{0}}{\alpha_{1}}\right\} .
\end{aligned}
$$

The motion in the small vicinity of the fixed points is stable (provided the phase $0<\phi_{s}<\pi / 2$ ) with the same synchrotron frequency $\Omega_{s}$ proportional to $\alpha_{0}$,

$$
\Omega_{s}^{2}=\frac{e V \alpha_{0} \omega_{0} \omega_{\mathrm{rf}}}{2 \pi E_{0}} \sin \phi_{s}
$$

Hence, generally there are two bunches within the rf wave length centered at the two FPs. The second bunch (centered at the 2nd FP) moves ahead of the 1st bunch (centered at the 1st FP) and, in the region with the dispersion $D_{x}$, is shifted horizontally by $\Delta x=-\left(\alpha_{0} / \alpha_{1}\right) D_{x}$ relative to the centroid of the first bunch. For the nominal lattice, where $\alpha_{0} / \alpha_{1}$ is large, the shift is larger than the beam pipe aperture, and the phase plane looks like the upper plot in Fig. 2. For sufficiently small $\alpha_{0}$, the second stable point may be within the physical aperture and two bunches can be stable within one rf wave length.

The ratio $\alpha_{0} / \alpha_{1}$ defines not only the energy shift of the bunches but also the energy acceptance for each bunch. It has to be large compared to the rms energy spread $\delta_{0}$, say, $\alpha_{0} / \alpha_{1}>\simeq 10 \delta_{0}$. Parameter $\alpha_{1}$ is given, mostly, by sextupoles and, as in the example below, $\alpha_{1} \simeq 0.05$. Therefore,
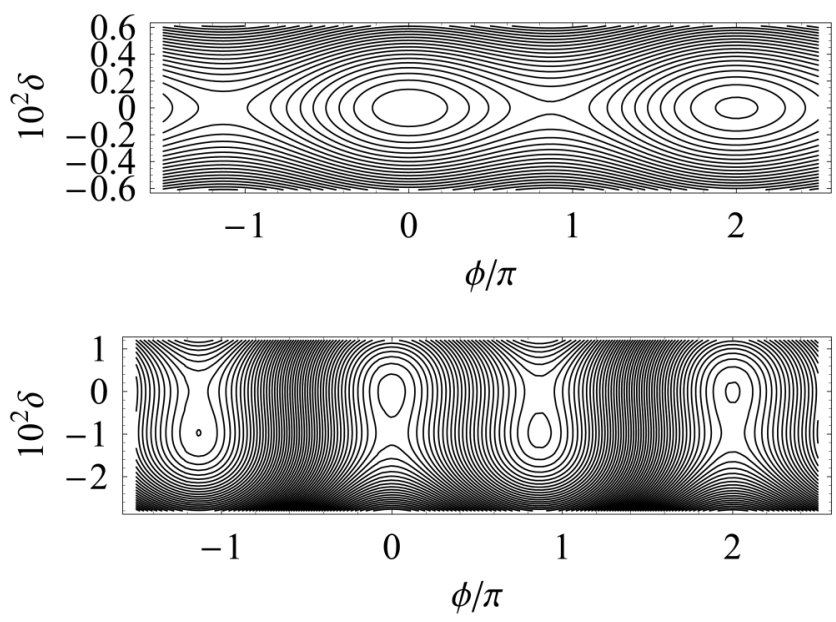

FIG. 2. Phase plot for the Hamiltonian $H(\delta, \zeta)=\alpha_{0} \delta^{2} / 2+$ $\alpha_{1} \delta^{3} / 3-\lambda(\sin [\phi-\zeta]-\sin [\phi])-\lambda \zeta \cos [\phi]$. Parameters are $\lambda=2.0 \times 10^{-7}, \quad \alpha_{1}=5.5 \times 10^{-2}, \quad$ and $\quad \alpha_{0}=5.0 \times 10^{-2}$ (above), and $\alpha_{0}=5.34 \times 10^{-4}$ (bottom). Note the different vertical scale for two plots. to allow two bunches per rf bucket, the lattice has to be designed to give small but not too small $\alpha_{0}$, only by an order of magnitude smaller than the nominal $\alpha_{0}=2.4 \times$ $10^{-3}$

The PEP-II rings have quite small $\delta, \delta_{0}=6.1 \times 10^{-4}$ for HER and $\delta_{0}=7.7 \times 10^{-4}$ for LER. Another specific advantage of the PEP-II is that it operates using the high repetition rate of injection, which relaxes requirements for the dynamic aperture and energy acceptance.

\section{THE LONGITUDINAL WAKE}

We model the wake field of a pointlike bunch for the LER PEP-II B-factory adding contributions of the experimentally measured modes of six rf cavities, resistive wall, and the inductive components of the ring. The later are described by the inductivelike model with the wake $W_{L}^{\delta}$ [7],

$$
W_{L}^{\delta}(z)=\frac{L}{\sqrt{\pi z a^{3}}}\left(1-\frac{z}{a}\right) e^{-z / a},
$$

where the inductance $L=80 \mathrm{nH}$ corresponds to the estimated inductance of the ring and the parameter $a=$ $0.285 \mathrm{~cm}$ is chosen to reproduce the total loss factor of the small vacuum components of the ring. The wake $W_{L}^{\delta}>$ 0 corresponds to energy loss.

The wake $W(z)$ for pointlike particles convoluted with the Gaussian bunch with rms bunch length $\sigma_{0}=0.8 \mathrm{~mm}$ is shown in Fig. 3.

\section{BUNCH PROFILE}

The equilibrium steady-state longitudinal bunch profile is given by the Haissinski solution. For $\phi_{s} \simeq \pi / 2$, the FPs are well separated, $\Delta z \simeq \lambda_{\text {rf }} / 2$. A small tune shift for the trailing bunch at the first FP is generated by the leading bunch at the second FP and, with nonzero chromaticity, by the energy offset of two bunches. However, because the

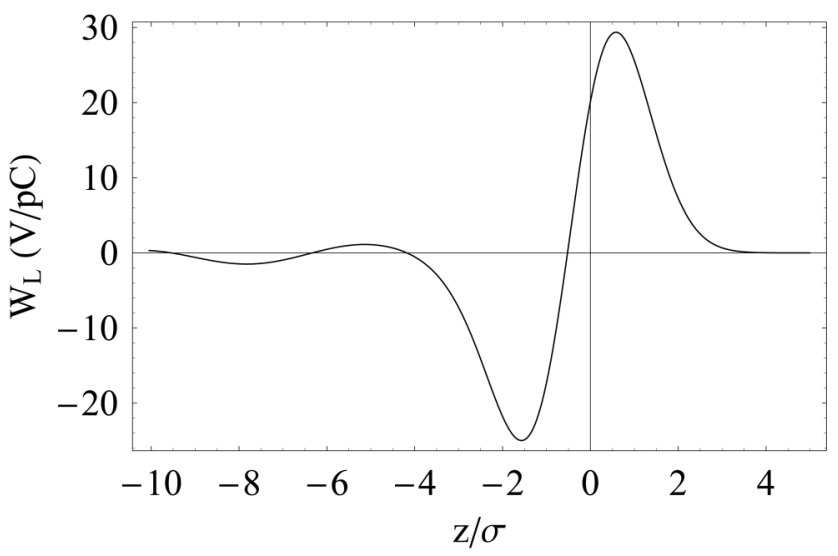

FIG. 3. The longitudinal wake field obtained by convolution of the $W \delta(z)$ with the $\sigma=8 \mathrm{~mm}$ Gaussian distribution. 


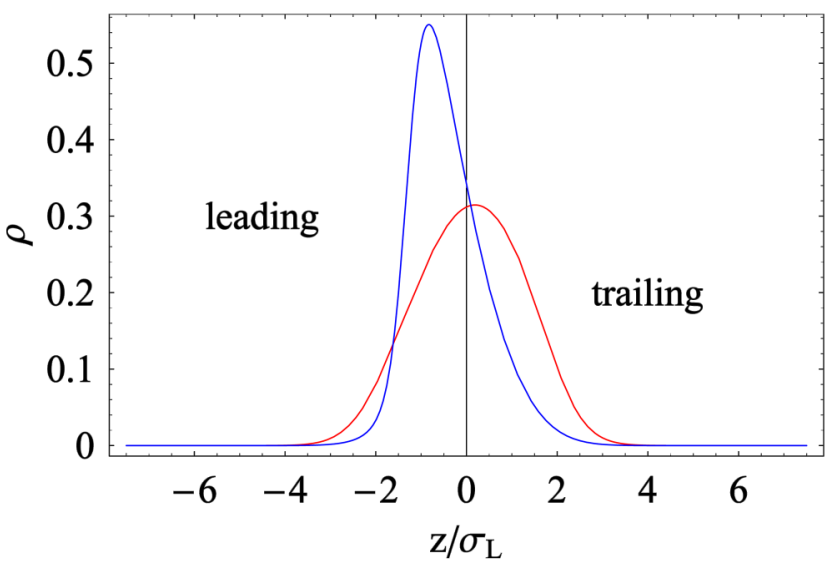

FIG. 4. (Color) An example of the bunch profiles for the leading (the bunch centered at the 2nd FP, blue line) and trailing bunch (centered at the $1 \mathrm{st} \mathrm{FP}$, red line). Parameter $\alpha_{0}=0.8 \times 10^{-3}$. The zero current $\sigma_{0}=1 \mathrm{~cm}$, the bunch current $I_{B}=2.5 \mathrm{~mA}$.

broadband wake at large $z_{2} \gg \sigma_{0}$ is small, the shift can be neglected. For well-separated bunches, quantum diffusion between FPs is exponentially small. For realistic bunches $\sigma_{l} \ll \Delta z$, and the bunch profile $f(z)$ can be written as Haissinski distribution

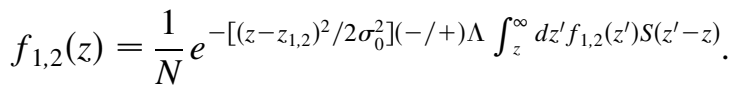

Here $S(z)$ is given by the longitudinal wake $W^{\delta}(z)$, $S(z)=\int_{0}^{z} W^{\delta}\left(z^{\prime}\right) d z^{\prime}, S(z)=0$ for $z<0$, and

$$
\Lambda=\frac{N_{b} r_{e}}{2 \pi R \gamma \alpha_{0} \delta_{0}^{2}},
$$

where $N_{b}$ is the number of particles per bunch, $r_{e}$ is the classical electron radius, $\gamma$ is the relativistic factor, $\delta_{0}$ is the rms relative energy spread, and $2 \pi R$ is the ring circumference. The normalization constant $N$ is given by the condition $\int f_{1,2}(z) d z=1$. The energy spread is defined by synchrotron radiation and is the same for both bunches, as well as parameters $\sigma_{0}$ and $\Lambda$.

The bunch profile depends only on two parameters: $\Lambda$ and the zero current rms bunch length $\sigma_{0}$. Variation of $\alpha_{0}$ at the constant $\sigma_{0}$ implies simultaneous variation of the $\mathrm{rf}$ voltage.

The terms depending on wakes enter in Eq. (6) with opposite signs which is the result of the opposite slope of the rf voltage at the FPs. Therefore, the dynamics of the leading bunch is the same as the dynamics of a bunch in the lattice with the negative momentum compaction factor.

The potential well distortion makes the first (leading) bunch shorter and the second (trailing) bunch longer. The trailing bunch has the usual tilt forward while the leading bunch tilts backward (Fig. 4) and has smaller rms due to the negative sign of the wake term in Eq. (6). Variation of the rms bunch length with current is shown in Fig. 5. In this study (and the simulations of the bunch stability below) we use the wake $W(z)$ convoluted with $2 \mathrm{~mm}$ Gaussian distribution. The bunch lengthening for typical mostly inductive wakes in the case $\alpha>0$ is replaced, as it is claimed usually, by the bunch shortening for $\alpha<0$ and low bunch currents. However, at higher bunch currents simulations show that bunch lengthening takes place in both cases. The wake field and parameters used in calculations are taken for the impedance model and PEP-II parameters.

\section{MICROWAVE INSTABILITY}

Parameter $\Lambda$ is inversely proportional to $\alpha_{0}$. Therefore, the main objection to the quasi-isochronous lattice is based on the expectation of the substantially reduced threshold of the microwave instability. We defined the microwave threshold using the Fokker-Planck solver [8] developed by one of the authors (S.N.) The threshold of instability

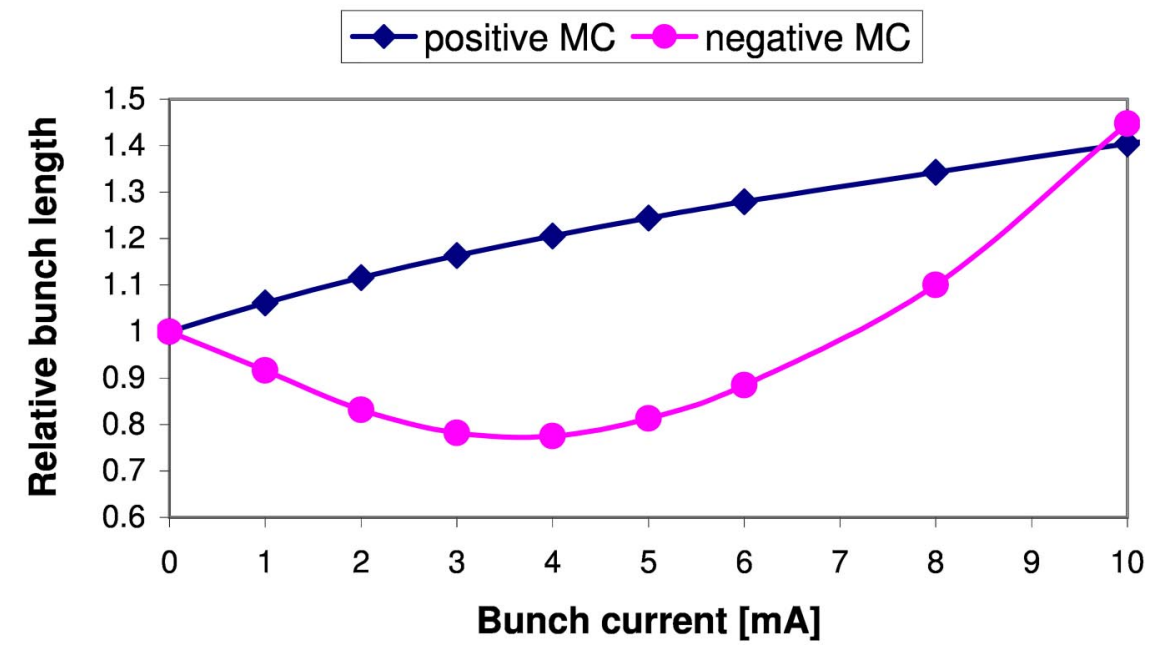

FIG. 5. (Color) Bunch lengthening vs bunch current for positive and negative momentum compaction (MC) factors. 


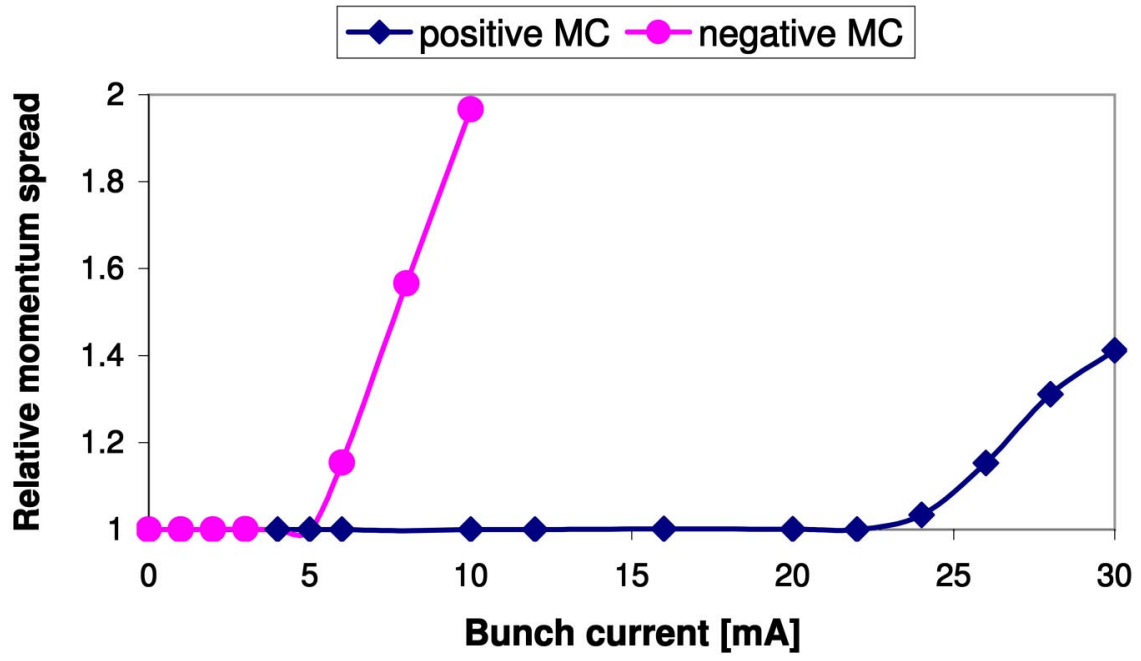

FIG. 6. (Color) Energy spread vs beam current for positive and negative momentum compaction factors.

is indicated by the growth of the energy spread for the bunch current $I>I_{\mathrm{th}}$. Dependence of the energy spread on current is shown in Fig. 6 for positive and negative momentum compaction (MC) factors for the same wake. As the figure shows, the $\propto 20 \mathrm{~mA}$ threshold for $\alpha_{0}>0$ is reduced to $5 \mathrm{~mA}$ for $\alpha_{0}<0$. However, even $5 \mathrm{~mA}$ bunch current is by a factor of 2 higher that the present PEP-II bunch current and may be acceptable.

\section{LONGITUDINAL HEAD-TAIL INSTABILITY}

The longitudinal head-tail (LHT) instability is, probably, the main problem of the low-momentum compaction lattices. The growth rate of instability [6]

$$
\frac{1}{\tau}=-\frac{1}{3 T_{0}} \frac{\alpha_{1}}{\alpha_{0}}\left(\frac{\sigma_{z}}{E}\right)\left(\frac{d \Delta E}{d \sigma_{z}}\right)
$$

where $T_{0}$ is revolution period, $\sigma_{z}$ is the rms bunch length, $\Delta E$ is the energy loss per turn per bunch, $E$ is the beam energy. The growth rate depends on the variation of the energy loss with the bunch length. For the case of $\alpha_{1} \simeq \alpha_{0}$ the growth rate is low and the instability is usually suppressed by the synchrotron radiation (SR) damping. The situation is different for our case with the anomalous large ratio $\left(\alpha_{1} / \alpha_{0}\right)$. The growth rate in this case may exceed the SR damping. As an example, the extreme case of the strong instability is shown in Fig. 7. The bunch dynamics for the $2 \mathrm{~mA}$ bunch current is shown in the phase plane $(z, \delta)$. Time indicated in the figure is in number of turns. Initially, the bunch is located at the FP corresponding to momentum compaction $\alpha<0$. After $t>600$ turns, the bunch splits in halves. The same simulations with the bunch initially located at the FP corresponding to $\alpha>0$ shows only stable motion. Results are obtained using the direct solution of the Fokker-Planck equation [8].

To study the longitudinal HT instability, we carried out two type of simulations. In the simple simulations, we calculate trajectories of four particles solving with MATHEMATICA equations of motion. That allows study of the dynamics of the system including quantitative result for the emittance variation. Equations include synchrotron oscillations and interaction between particles proportional to the wake convoluted with $\sigma=8 \mathrm{~mm}$ Gaussian bunch,

$$
\frac{d \zeta_{i}}{d \tau}=-\delta_{i}\left(1+\epsilon \delta_{i}\right)
$$

$$
\frac{d \delta_{i}}{d \tau}=\zeta_{i}-\frac{1}{4} \sum_{j} I_{\mathrm{bunch}} W\left[\zeta_{i}-\zeta_{j}\right]
$$

where $(i, j)=1,2,3,4$, the dimensionless $\zeta$ is the distance of a particle from the bunch center in units of the rms bunch $\sigma$ and $\delta$ is relative energy offset $\Delta E / E$ in units of the relative $\mathrm{rms}$ energy spread $\delta_{0}, \tau=\omega_{s} t$, and $\epsilon=$ $\left(\alpha_{1} / \alpha_{0}\right) \delta_{0}$. Note that in this units one revolution period $T_{0}=2 \pi$. In the simulations we used LER parameters, the synchrotron period equal to 56 revolution periods, $\alpha_{0}=$ $5.34 \times 10^{-4}, \quad \alpha_{1}=5.54 \times 10^{-4}, \quad \delta_{0}=7.7 \times 10^{-4}$, and $\sigma=8 \mathrm{~mm}$.

Trajectories were calculated with initial conditions $\zeta_{i}=$ $-0.33,-0.27,0.27,0.33, \delta_{i}=0$ for the particles initially located at the FP corresponding to $\alpha<0$ for the time interval up to 700 synchrotron periods. Results for the bunch current $I_{\text {bunch }}=0.5 \mathrm{~mA}$ clearly show that the system in unstable although the growth rate is small.

We tried to understand whether the instability can be compensated. The LHT instability is a peculiar one: although it is a single-bunch instability, the cause of the instability is the variation of the energy loss per bunch coupled to the motion of the bunch centroid. Therefore, it seems that the instability might be stabilized by the longitudinal feedback system (FB).

We model the FB generating a buffer $\zeta_{1}, \ldots, \zeta_{56}$ which stores positions of the bunch centroid for the last of 56 

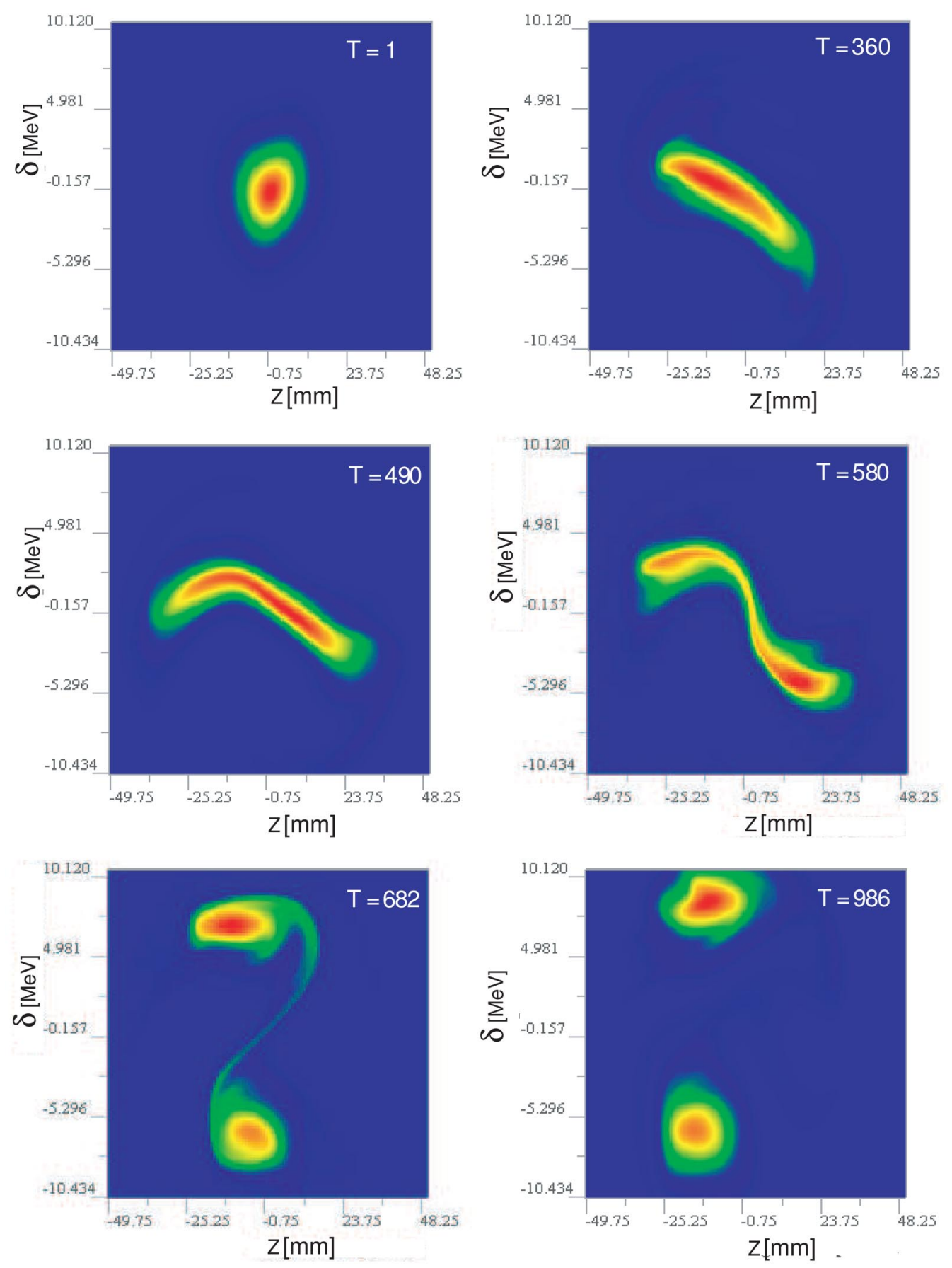

FIG. 7. (Color) Dynamics of the head-tail single-bunch instability is shown in the phase plane $(z, \delta)$ for $2 \mathrm{~mA}$ bunch current. Time in the number of turns is indicated in the figure. At $t>600$ turns, the bunch splits in halves.

revolutions per synchrotron period. The data are interpolated as the sum of

$$
\begin{aligned}
\zeta_{f}\left(\tau_{k}\right)= & a_{0}+a_{1} \sin \left(\nu \tau_{k}+\phi_{1}\right)+a_{2} \sin \left(2 \nu \tau_{k}+\phi_{2}\right) \\
& +a_{3} \sin \left(3 \nu \tau_{k}+\phi_{3}\right)
\end{aligned}
$$

where $\tau_{k}=\tau-(k-1) T_{0}, k=1, \ldots, 56$, and $a_{j}, j=$ $0,1,2,3$ and $\phi_{j}, j=1,2,3$ are determined as the fitting parameters. Then, the kick $\delta_{i} \rightarrow \delta_{i}+K$ is applied to each of four tracking particles where $K=0.1\left[d \zeta_{f}(\tau) / d \tau\right]_{\tau=\tau_{1}}$ is proportional to the derivative of the fitting function. The coefficient 0.1 was determined to give the best damping. The result of tracking for each of the particles with the FB on is shown in Fig. 8. The results seem encouraging: the amplitude of the oscillations for each particle remain stable for 700 synchrotron periods.

More elaborate simulations used the Fokker-Planck solver developed to study microwave instability. 

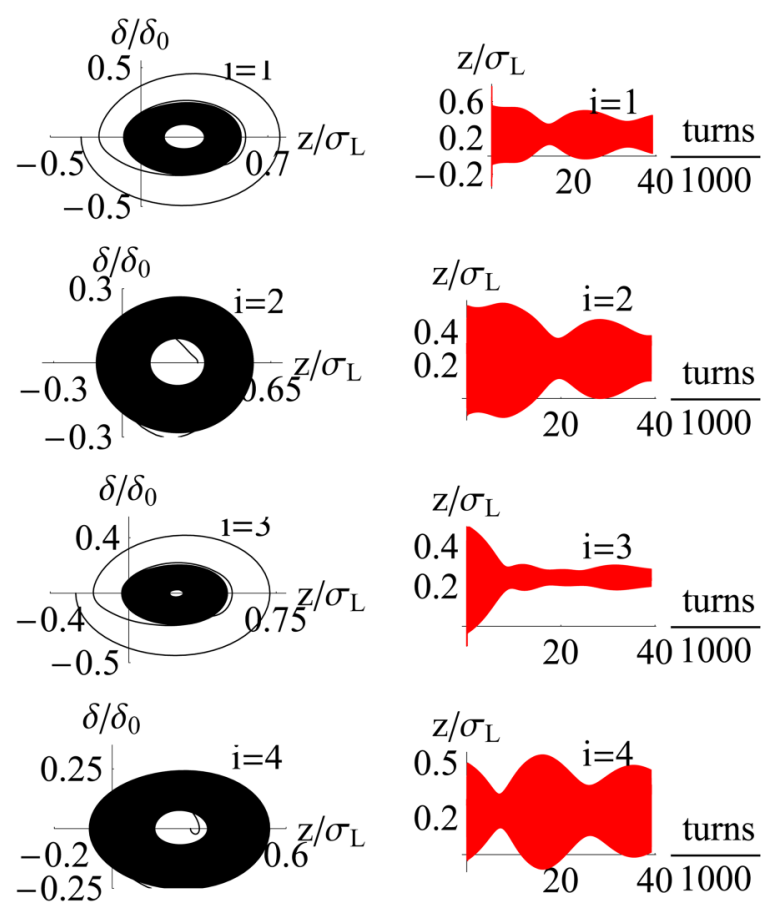

FIG. 8. (Color) Tracking of four particles with the feedback on. The $i$ th row shows the trajectory in the phase plane (on the left) and the displacement $\zeta_{i}(\tau)$ (on the right) for the $i=1,2,3,4$ particle. The trajectory after one or two turns finds the fix point and then remains stable for 700 synchrotron periods.
Feedback is introduced in the same way as described above. Results in Fig. 9 show that the beam centroid is indeed stable. However, the time dependences of the emittance and the energy spread do not confirm conclusion of the simplified simulations. Results of the simulations with the Fokker-Planck solver are shown in Fig. 10. Emittance and energy spread, stabilized initially as in the simplified simulations, slowly grow at a later time. Growth starts with the bunch deformation (see insert) which would later be followed by splitting of the bunch in halves as shown in Fig. 7. The difference of two simulations is apparently due to the difference in the models: the two-particle model does not include fluctuations which are included in the FokkerPlanck equation.

\section{SUMMARY}

The quasi-isochronous ring with the reduced momentum compaction factor allows us to have two bunches per $\mathrm{rf}$ bucket. It is tempting to increase the number of bunches per ring without increasing rf frequency. This paper presents a study of this possibility. We consider lattice design, bunch lengthening, and microwave and longitudinal head-tail instabilities. Other effects such as parasitic crossings and $\mathrm{rf}$ beam loading are also included in the consideration in [2]. The bunch lengthening and microwave instability seem to give weak constraints. However,

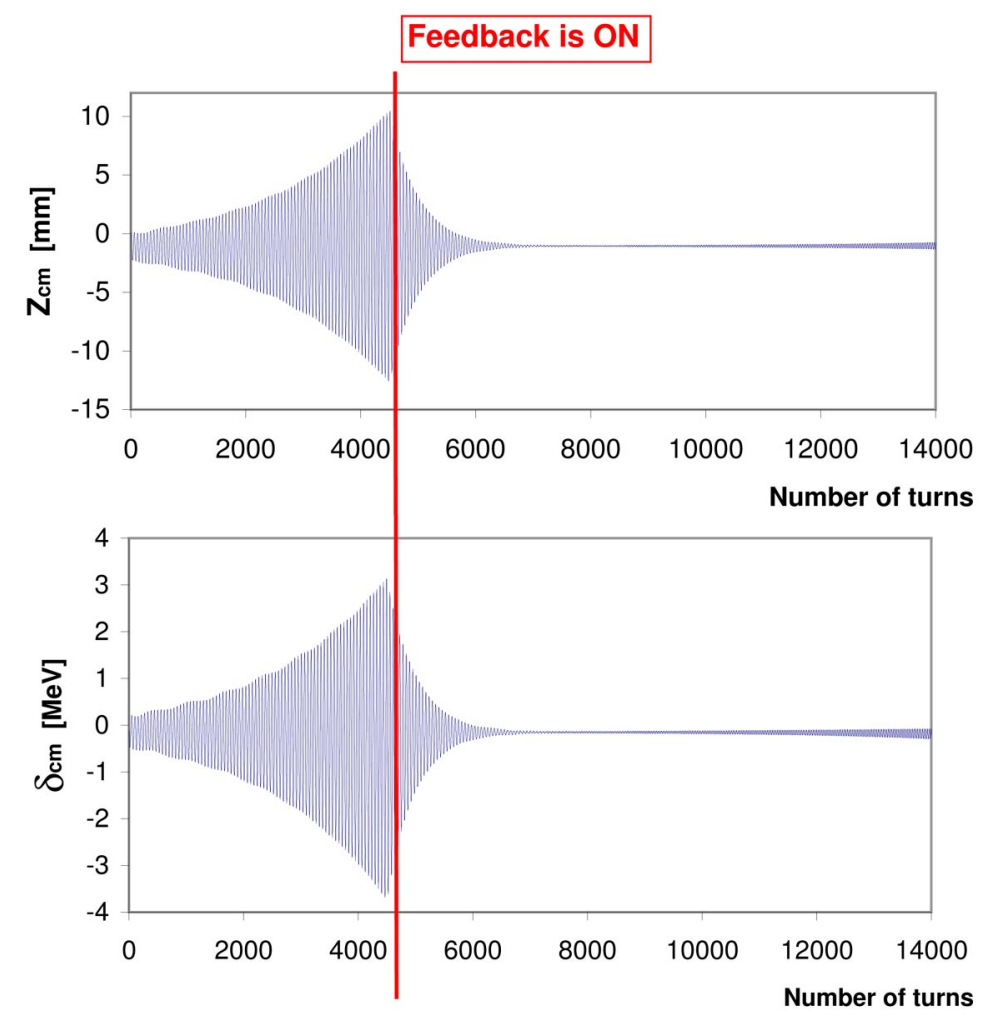

FIG. 9. (Color) Variation of the centroid offset $Z_{\mathrm{cm}}$ and the centroid energy $\delta_{\mathrm{cm}}$ in time. Results are from the solution of the FokkerPlanck equation with the feedback on. 


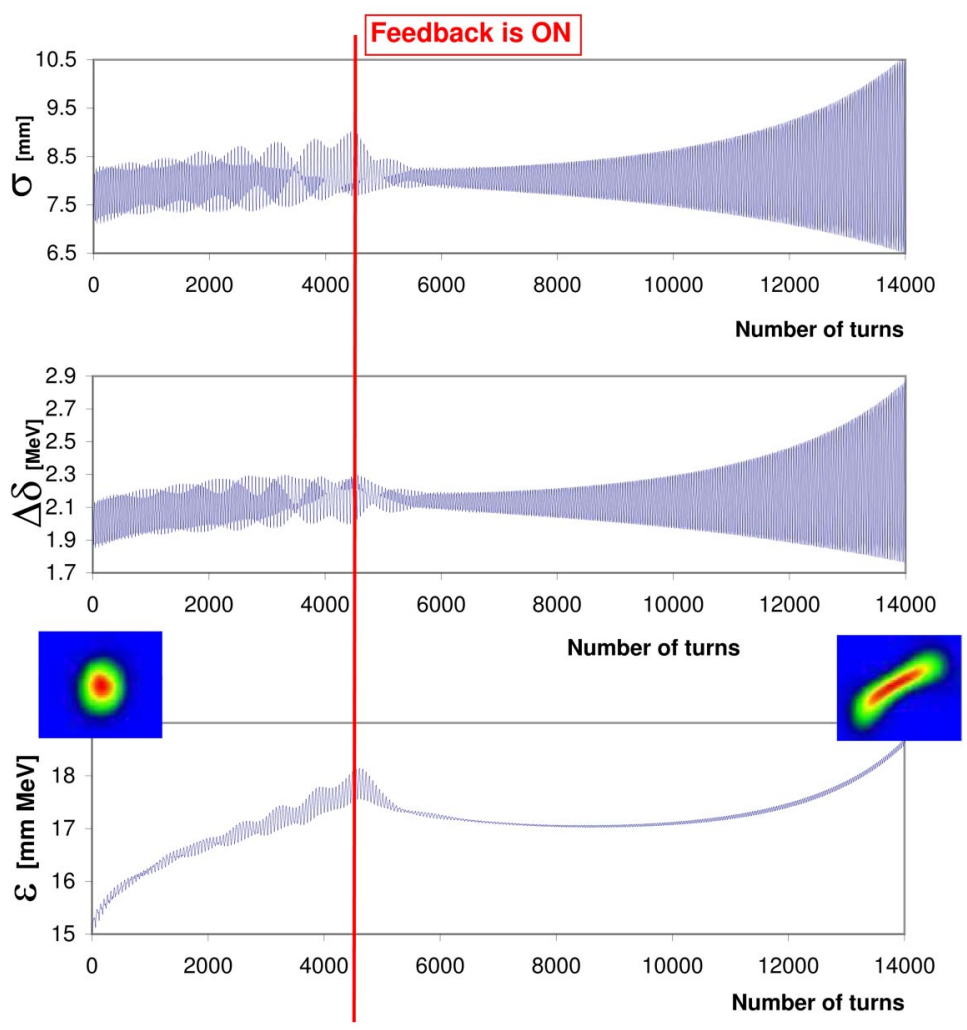

FIG. 10. (Color) Variation of the bunch length $\sigma$, the energy spread $\Delta \delta$, and emittance $\epsilon$ in time. Results are from the solution of the Fokker-Planck equation with the feedback on.

the longitudinal head-tail instability makes the beam unstable. That sets a serious limitation on the applicability of low-alpha lattices. We tried to utilize the peculiar feature of the instability, coupling to the motion of the bunch centroid, applying the rigid bunch dipole feedback system. We show that although there is a stabilizing effect of such feedback, we found that instability can not be fully stabilized although the growth rate of instability is small. Fluctuations make the particles in the second fixed point unstable. Therefore, the statement that there are two stable fix points in low-alpha lattices may be an illusion unless a longitudinal feedback system with a bandwidth proportional to the inverse bunch length is utilized.

\section{ACKNOWLEDGMENTS}

The authors thank Y. Nosochkov for his help with MAD. This work was supported by Department of Energy Contract No. DE-AC02-76SF00515.
[1] A. Gallo, P. Raimondi, and M. Zobov, in Proceedings of the 31st ICFA BD Workshop, SLAC 2003 (physics/ 0404020).

[2] S. Heifets and A. Novokhatski, Report No. SLAC-PUB11467, 2005.

[3] J. B. Murphy and S. L. Kramer, Phys. Rev. Lett. 84, 5516 (2000).

[4] W. J. Corbett, M.H.R. Donald, and A. A. Garren, in Proceedings of the Particle Accelerator Conference, San Francisco, CA, 1991 (Report No. SLAC-PUB-5538, LBL30669, 1991).

[5] G. Wustefeld et al., in Proceedings of the BESSY Low alpha Optics, ICFA Mini-Workshop SBSR-05, Frascati, 2005.

[6] Handbook of Accelerator Physics and Engineering, edited by A.W. Chao and M. Tigner (World Scientific, Singapore, 1998).

[7] S. Heifets and A. Chao, Report No. SLAC-PUB-8398, 2000.

[8] A. Novokhatski, Report No. SLAC-PUB-11251, 2005. 